Journal of Bionic Engineering 7 (2010) 112

Copyright (C) 2010, Jilin University. Published by Elsevier Limited and Science Press. All rights reserved.

doi: 10.1016/S1672-6529(09)60197-9

\title{
Notice of Modification
}

\section{Dapeng Yang}

State Key Laboratory of Robotics and System, Harbin Institute of Technology, Harbin 150001, P. R. China

Dear readers,

In the paper "An Anthropomorphic Robot Hand Developed Based on Underactuated Mechanism and Controlled by EMG Signals", Journal of Bionic Engineering, 2009, 6, 255-263, by Dapeng Yang et al., we adopted an experimental method already developed by the DLR (German Aerospace Center). Unfortunately, we did not cite their main works properly at that time. We sincerely apologize for this mistake to Dr. Castellini, Dr. van der Smagt and other coauthors.

More details about the use of OttoBock's electrodes, the use of a Support Vector Machine for classification, the data analysis and use of the terms "session" and "group", and the presentation of accuracy results can be found in these papers by Castellini et al:

[1] Castellini C, van der Smagt P, Sandini G, and Hirzinger G. Surface EMG for Force Control of Mechanical Hands. Proceedings of International Conference on Robotics and Automation, Pasadena, CA, USA, 2008, 725-730.

[2] Castellini C, van der Smagt P. Surface EMG in Advanced Hand Prosthetics. Biological Cybernetics, 2009, 100, $35-47$. 